\title{
Visual Data Mining for Multimedia Databases
}

\author{
Karthikeyan Chinnusamy, A. Rajaraman
}

\begin{abstract}
With computers and communication dominating technology in different fields, the need to look for media-based information processing, MBIP-rather than data-based information processing, DBIP- is increasingly being felt and this is compounded by the explosive developments in cellular communication, which brought computing and interaction on the move. The basis is to explore possibilities of using conventional data-mining approaches with visualization and object orientation so that human interaction is easier.

Data Mining involves exploring databases to try and discover data relationships which are not explicitly stored with in the databases. Traditional techniques involve statistical analysis, clustering and pattern matching. Many current efforts are underway to integrate visualization in to this process.

Visual data mining is a novel approach to data mining. The aim is to combine traditional data mining algorithms with information visualization techniques to utilize the advantages of both approaches. The utilization of both automatic analysis methods and human perception/understanding promises better and more effective data exploration. Visualization is a key process in visual data mining. Here the focus is on the presentation of all aspect of multimedia objects, their identification, their analysis and relationships.
\end{abstract}

Keywords--- Visual Data Mining, Multimedia Databases, Visual data, Self-Organizing Maps.

\section{INTRODUCTION}

Current trends in data mining include mining unstructured data such as text, voice, and video, mining data in distributed and heterogeneous databases, and mining the World-Wide Web (WWW) data to help electronic commerce sites [1]. Several prerequisite are required for successful data mining. The first is data preparation, as good data are essential for good mining. Data have to be scrubbed and cleaned, and in some cases warehoused. Inconsistencies and uncertainties must be eliminated. When data preparation step is completed, the next step is to determine the desired outcomes, such as clustering, associations, and the subsequent selection of appropriate tools to carry out mining .This is largely a trial and error process. Engineers improve their performance with experience. Applying the data mining tools and getting some results is less complex task than making sense out of the data. The first step is to carry out some actions based on the results to determine whether the mining effort was worthwhile.

\section{MOTIVATION FOR MULTIMEDIA AND VISUAL DATA MINING}

While the very largest data sets, i.e. data sets larger than $10^{8}$ bytes, are not easily accessible via graphical visualization methods, data visualization is often powerful tool for the exploration of data. In particular several visualization tools, which when used in conjunction with each other, form an extremely useful approach to visual data mining. Wegman discusses issues of computational complexity and data transfer as well as visual complexity. At some where between $10^{6}$ to $10^{7}$ bytes, analysis with complex algorithms on conventional desktop computing begins to fail. Similarly, data transfer over standard Ethernet begins to take an unfeasibly long amount of time. A second critical way-point occurs at data set size of about $10^{12}$. For small enough data set sizes data can be stored in memory or primary storage. For larger data sets, data must be stored on hard disks or secondary storage and accessed piecemeal. For massive datasets, data must be stored in robotic magnetic tape silos or tertiary storage. Access becomes orders of magnitude slower and focus shifts dramatically from statistical considerations to computational considerations [2]

For purposes of visual data mining, a critical question is what is the maximum number of data points we can process visually? After all, exploratory data analysis is traditionally graphically oriented. Such Questions must me answered in terms of the human visual system which clearly begins with finite number of cells in the eye, most importantly the high resolution foveal cones. Based on both psychometric experiments and anatomical considerations, somewhere around $10^{6}$ to $10^{7}$ observations appears to be the practical limit for visualization of data while massive data sets easily venture in to the range of $10^{12}$ bytes. The human eye has approximately $10^{7}$ cones implying that visualizing one observation per cone would optimistically put the upper limit of visual resolution at about $10^{7}$ observations. Thus, data mining as such cannot successfully exploit visualization for truly massive datasets (like multimedia data) without some modification of the raw data. Several approaches like thinning and binning to reduce the size of the data sets making visual analysis more feasible.

Multimedia data is typically has a complex structure and cannot readily be processed as a whole by the data mining algorithms. Image and video indexing and retrieval have always been an interesting research field. The advent of World Wide Web brought new challenges to computer vision and artificial intelligence community. Research in computer vision, artificial intelligence, databases, etc is taken to a large scale to address the problem of information retrieval from large repositories of images. Traditional pattern recognition and image analysis algorithms in vision and AI fields dealt with small sets of still images and did not scale well.

\footnotetext{
Manuscript received September 16, 2019.

Karthikeyan Chinnusamy, Veritas. (e-mail: karthikchinnusamy@ieee.org)

Dr.A. Rajaraman, Visiting Professor, Indian Institute Technology, Chennai. T.N, India. (e-mail: arraman_2000@yahoo.com)
} 
Whereas much of the previous work on data mining focused on mining relational databases. Object models are very popular for multimedia data, and therefore, we need to mine object databases to extract useful information from the large quantities of multimedia data. For machine learning and knowledge discovery multimedia mining brings new challenges as the data is extremely large in size, uses different modalities and has a rich internal structure. Required technologies include speech recognition, text mining, video and image analysis information retrieval and summarization.

\subsection{Multimedia Mining}

The progress made in the hardware technology allows today's computer system to store very large amounts of data. It is estimated that every year about 1Exabyte of data are generated, of which large portion is available in digital form. Even simple transactions of every day life are typically recorded by computers [9]. Usually many parameters are recorded resulting in multidimensional data with high dimensionality. Finding the valuable information hidden in them is however a difficult task. With this today's data management systems, it is only possible to view quite small portions of data. If the data is presented textually, the amount of data which can be displayed is in the range of some one hundred data items, but multimedia kind of data will contain data sets with millions of data items. For setting meaningful information out of that we need to explore the multimedia data. The exploration can be done with visual data mining techniques [6].Much of the bandwidth on the Internet is taken up by transmission of visual data. The bulk of this data is never analyzed or used because of lack of efficient and scalable techniques.

Visual data exploration aims at integrating the human in the data exploration process, applying its perpetual abilities to the multimedia data. Visualization is the key process in Visual data mining. Visualization technique can provide a clear and more detailed view on different aspect of data as well as results of automated mining algorithms. Here we will see the information content and information structure parts in which the information is organized.

Now the challenge is to mine data in the multimedia objects. One way is to extract relational data from multimedia data. The other is to mine object databases directly. First we need to identify the types of mining that could be carried out (like clustering, estimation and prediction. The recent advances in research on multimedia databases [11] enable the creation of large multimedia databases which can be queried in an effective way. These advances, in combination with the research in to multimedia database and advances in data mining in relational databases [2], created a possibility for the creation of multimedia data mining systems.

We could develop techniques to retrieve values of the attributes and then find some relationships between the values. We can write additional methods to extract certain values and then do some analysis. Visual data mining techniques proven to be of high value in exploratory data analysis and they have high potential for exploring large database, in particular when we have little is known about the data and the exploration goals are vogue. When we are using visual data mining the user is directly involved in the exploration process, shifting and adjusting the exploration goals can be automatically done.

In text mining, the retrieval of document can be done by specifying attributes or key values. Also we required to discover associations between the words and paragraphs. Since most of the available data mining tools support only relational databases (Structured data),we required to develop tools that can handle unstructured text data.[7],[8].

Image mining deals with mainly associations between different images from large image database. We have to find out unusual patterns in the images. We need to extract Metadata from images and then mining can be done on the metadata [10] [12]. The visualization techniques are very much useful in finding the unusual patterns in the images.

Video mining and audio mining are difficult compared to text mining and image mining. But one of the way is to convert the available information from video and audio database to text [13]. For this visual mining can be used. Using visual mining we can gain insight into unstructured data and can come up with new hypotheses. The verification of the hypotheses can also be done via visual data exploration but it may also be accomplished by automatic techniques from statistics or machine learning.

\section{VISUAL MINING TECHNIQUES \& RESULTS}

Many multimedia data sets consist of more than tree attributes and therefore, they do not allow a simple visualization as two-dimensional or three-dimensional plots. Since there is no simple mapping of the attributes to the two dimensions of the screen, more sophisticated visualization techniques are needed [3],[4]. This can be achieved by the following techniques.

\subsection{Self-Organizing maps (SOM)}

SOM provide a effective mechanism for preprocessing and organizing unstructured data. SOMs are able to extract groups of similar information objects and can be described as nonlinear projection from n-dimensional input space on to two dimensional visualization spaces [5],[14] .

\subsection{Progressive Resolution Refinement}

For effective and efficient discovery of patterns in multimedia databases, a multi-resolution strategy can be used .By first finding the patterns at a low resolution and preserving the search at a higher resolution with only the data selected in lower resolutions. The basic idea is quickly approximate patterns at a course level, and then eliminates false positives by verifying them at a higher resolution. This can be done by using image processing algorithms such as image enhancement, filtering and transformation.

\subsection{Geometrically Transformed Displays}

Geometrically transformed display techniques aim at finding interesting transformations of multidimensional data sets. 
The class of geometric display techniques includes techniques from exploratory statistics, such as scatter plot matrices [15], [16] and techniques which can be subsumed under the term projection pursuit [17]. Other geometric projection techniques include Prosecution Views [18], [19], Hyper slice [20], and the well-known Parallel Coordinates visualization technique [21]. The parallel coordinate technique maps the k-dimensional space onto the two display dimensions by using $\mathrm{k}$ equidistant axes which are parallel to one of the display axes. The axes correspond to the dimensions and are linearly scaled from the minimum to the maximum value of the corresponding dimension. Each data item is presented as a polygonal line, intersecting each of the axes at that point which corresponds to the value of the considered dimensions

\section{CONCLUSION}

In this paper we have proposed the visual data mining techniques for the multimedia database. Visual data exploration process, which applies its perpetual abilities to the multimedia data. Visualization techniques provide a clear and more detailed view on different aspect of data as well as on results, if automated mining algorithms.

\section{REFERENCES}

1. P.Adrian and D.Zaninge ,"Data Mining”,Addison Wesley,1996.

2. U.M.Fayyad, G.Piatetsky -shapiro, P.Smyth, Uthurusamy, "Advances in Knowledge Discovery and Data Mining", AAAI/MIT Press,1996,pp1-36.

3. Abello.J,Korn.J," A System for Visualizing massive multidigraphs", Transaction on Visualization and Computer Graphics,2001.

4. Stasko.J, Zhang. E," Focus + Context Display and Navigation Techniques for Enhancing Radial SpaceFilling Hierarchy Visualization", IEEE information Visualization, 2000, Salt Lake City, UT, 2000, pp-57-65.

5. Kreuseler.M, Lopez.N, Schumann.H, "A Scalable frame work for information Visualization", Proc. Inter Vis'2000,Salt Lake city, 2000, pp-27.

6. Daniel A.Keim," Information Visualization and Visual Data Mining", IEEE Transaction on Visualization and Computer Graphics, Vol 7,No 1,Jan-Mar 2002,pp 100107.

7. R.Feldmand, I.Dagan, "Knowledge Discovery in Textual Databases (KDT)",In Proc. $1^{\text {st }}$ Int .Conf Knowledge discovery and Data mining, Montreal, Canada, Aug1995,pp112-117.

8. R.Felman and H.Hirsh, " Mining associations in text in the presence of background Knowledge", In Proc. $2^{\text {nd }}$ Int. Conf Knowledge Discovery and Data Mining, Portland, Oregon, Aug 1996,pp343-346.

9. O.Etzionii, "The Worldwide Web: Quagmire or goldmine?", Communication of ACM, 39:65-68,1996.

10. U.M.Fayyad and P.Smyth, " Image database exploration: Progress and Challenges", In Proc. Knowledge Discovery in Databases workshop, Washington, D.C,1993, pp-14-27.

11. M.Flickner, H.Sawhey, W.Niblack and et al ,"Query by image and video content: The QBIC System", IEEE Computer, September, 1, 1995, pp 23-32.

12. K.Hirata, S.Mukherjea et al, "Integration of image matching and classification for multimedia navigation" Multimedia tools and Applications, 11, 2000, pp 295 309.
13. M.G.Cenruti,C.Anken,A.D.Linans S.H.Rubin," Application of High performance Knowledge-based technology" In. proc. Of the 2000 IEEE International Conference on Systems, Man and Cybernetics (SMC 2000), Oct 2000,pp1965-1971.

14. Matthias Kreuseler and Heidrun Schumann," A Flexible Approach for Visual Data Mining", IEEE Transactions On Visualization And Computer Graphics, Vol. 8, No. 1, January-March 2002

15. D.F. Andrews, "Plots of High-Dimensional Data, ${ }^{\circ}$ Biometrics, vol. 29, pp. 125-136, 1972.

16. W.S. Cleveland, "Visualizing Data", Summit, N.J.: Hobart Press, 1993.

17. P.J. Huber, "The Annals of Statistics, ${ }^{\circ}$ Projection Pursuit, vol. 13, no. 2, pp. 435-474, 1985.

18. G.W. Furnas and A. Buja, "Projections Views: Dimensional Inference through Sections and Projections, ${ }^{\circ}$ J. Computational and Graphical Statistics, vol. 3, no. 4, pp. 323-353, 1994.

19. R. Spence, L. Tweedie, H. Dawkes, and H. Su, "Visualization for Functional Design, ${ }^{\circ}$ Proc. Int'l Symp. Information Visualization (InfoVis '95), pp. 4-10, 1995.

20. J.J. van Wijk and R.D. van Liere, "Hyperslice, ${ }^{\circ}$ Proc. Visualization '93, pp. 119-125, 1993.

21. A. Inselberg and B. Dimsdale, "Parallel Coordinates: A Tool for Visualizing Multi-Dimensional Geometry”, Proc. Visualization '90, pp. 361-370, 1990. 